\title{
Analysis of Candidate Waveforms for Integrated Satellite-Terrestrial 5G Systems
}

\author{
Arunprakash Jayaprakash*, Hongzhi Chen*, Pei Xiao*, Barry G. Evans*, Yingnan Zhang ${ }^{\dagger}$, Jing Yuan $\mathrm{Li}^{\dagger}$ \\ and Adegbenga B. Awoseyila* \\ ${ }^{*}$ Home of 5G Innovation Centre, Institute for Communication Systems,University of Surrey, Surrey GU2 7XH, U.K \\ *Email: \{a.jayaprakash, hongzhi.chen, p.xiao, b.evans, a.awoseyila\}@ surrey.ac.uk \\ ${ }^{\dagger}$ CAST Xi'an, China, Email: 66972515@qq.com
}

\begin{abstract}
Satellites will play an indispensable part in $5 \mathrm{G}$ roll out and the common use of new radio (NR) air interface will enable this. Satellite-terrestrial integration requires adaptations to the existing NR standards and demands further study on the potential areas of impact. From a physical layer perspective, the candidate waveform has a critical role in addressing design constraints to support non-terrestrial networks (NTN). In this paper, the adaptability of frequency-localized orthogonal frequency division multiplexing (OFDM)-based candidate waveforms and solutions are discussed in the context of physical layer attributes of non-linear satellite channel conditions. The performance of the new air interface waveforms are analysed in terms of spectral confinement, peak-to-average power ratio (PAPR), power amplifier efficiency, robustness against non-linear distortions and carrier frequency offset (CFO).
\end{abstract}

Index Terms-5G, satellite-eMBB, satellite-IoT, f-OFDM, WOFDM, TWTA

\section{INTRODUCTION}

$5 \mathrm{G}$ wireless communication systems aim to cover a number of heterogeneous use cases with specific service requirements in a cost effective manner that assures high quality of service (QoS). With the recent advancement in satellite technology such as the emergence of high throughput satellites and nongeo-stationary-orbit (NGSO) satellite constellation, an integrated satellite-terrestrial solution aims to meet the comprehensive and diverse service requirements of $5 \mathrm{G}$ such as maintaining a global network, ubiquitous coverage especially in sparsely populated areas, high throughput content distribution under high mobility, back-haul solution for massive number of devices, ultra reliability etc [1], [2]. In this scenario of satelliteterrestrial integrated 5G, further analysis and evaluation are required on the adaptation of the defined 3GPP 5G standards, which are identified in [3]. In this paper, we address the challenges and adaptations required on the physical layer standards and analyse the candidate waveforms for air interface under the new scenario. The major impairments that incur performance degradation in the physical layer aspects of the converged scenario are high non-linear distortion by the power amplifier, power-limited link budget and phase variations due to high mobility. Classical OFDM cannot overcome these challenges due to its large out of band (OOB) emission, high peak-toaverage power ratio (PAPR) and poor frequency localization. In order to mitigate these problems, new frequency-localized waveforms have been proposed, such as filtered-OFDM (f-
OFDM) [4], [5], windowed-OFDM (W-OFDM) [6], [7], filterbank multi-carrier (FBMC) [8], [9], generalized frequency division multiplexing (GFDM) [10], [11], universal filtered multi-carrier (UFMC) [12], [13] and their modified versions [14]. Among these, f-OFDM and w-OFDM preserve the receiver structure of OFDM and are well localized in frequency. Hence these are recommended by $3 \mathrm{GPP}$ as the waveforms for the new-radio (NR) in order to preserve the advantages of OFDM as well as maintain backward compatibility. The PAPR of the waveforms can be further reduced by DFT spreading and hence DFT-spread-f-OFDM (DFT-s-f-OFDM) and DFTspread-W-OFDM (DFT-s-W-OFDM) are also considered in the present investigation.

The remaining of the paper is organized as follows. A brief overview on OFDM based candidate waveforms is presented in Section II. In section III, the main challenges and factors which degrades the transmission-performance in the integrated satellite-terrestrial communication scenario are outlined. The numerical analysis and simulation results are presented in Section IV and conclusions are drawn in section V.

\section{FREQUENCY-LOCALIZED OFDM BASED CANDIDATE WAVEFORMS}

A brief overview of the frequency localized candidate waveforms: W-OFDM and f-OFDM is provided in this section. Orthogonal multi-carrier waveforms can generally be represented as

$$
s(n)=\sum_{k=-\infty}^{+\infty} \sum_{m=0}^{M} d_{k, m} p(n-k M) e^{(j 2 \pi m F(n-k M))}
$$

where $d_{k, m}$ represents the actual constellation points or DFT spread version of the actual constellation points, modulated at $m$-th sub-carrier and $k$-th symbol index, $p(n)$ denotes the prototype filter and $F$ is the sub-carrier spacing. The OOB suppression of the waveform can be further enhanced by passing the signal through a band-pass filter with impulse response, $f(n)$, to obtain

$$
y(n)=s(n) * f(n)
$$

where $*$ denotes linear convolution. The frequency localized OFDM based candidate waveforms are differentiated based on 
the selection and design of the two filters $p(n)$ and $f(n)$. The application of $p(n)$ and $f(n)$ through time-domain windowing and filtering respectively corresponds to W-OFDM and f-OFDM transmission schemes. The generic block diagram of the OFDM based transceiver [15] for the candidate waveforms is shown in Fig. 1.

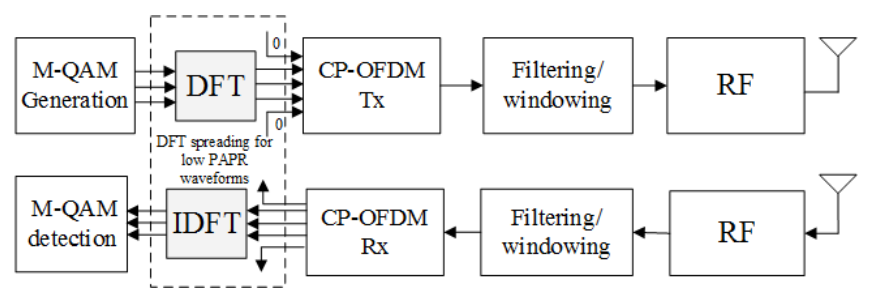

Fig. 1. Transceiver block diagram of OFDM based orthogonal waveform

\section{A. $f$-OFDM}

An f-OFDM transmitter first synthesises the conventional CP-OFDM signal using IFFT operation and then inserts the cyclic prefix. The prototype pulse $p(n)$ is the rectangular pulse as in the traditional CP-OFDM. The f-OFDM signal is then generated by filtering the OFDM signal with a bandpass filter $f(n)$ whose passband matches with the used subcarriers. A low-pass prototype filter is designed to suppress OOB emission and is generated as the product of ideal lowpass filter $f_{l}(n)$ with sinc impulse response and a truncation window $c(n)$. The normalized filter coefficients are generated as

$$
f(n)=\frac{c(n) \cdot f_{l}(n)}{\sum c(n) \cdot f_{l}(n)}
$$

where

$$
f_{l}(n)=\operatorname{sinc}\left((W+2 \times \delta w) \times \frac{n}{N}\right),
$$

$c(n)=\left(0.5\left(1+\cos \left(\frac{2 \pi n}{L-1}\right)\right)\right)^{0.6},-\left\lfloor\frac{L}{2}\right\rfloor \leq n \leq\left\lfloor\frac{L}{2}\right\rfloor$

Here, $W$ represents the number of used sub-carriers, $\delta w$ is a design parameter which accounts for the frequency roll-off at the pass-band edges. $L-1$ and $N$ denote filter order and FFT size respectively. The filter coefficients of the band-pass filter are generated by frequency shifting the ideal low-pass filter coefficients to the appropriate central frequency. The received signal is passed through the corresponding matched filter $f^{*}(-n)$ and then fed to the conventional OFDM receiver.

\section{B. $W$-OFDM}

In W-OFDM, a pulse with improved frequency response is used as $p(n)$ instead of the rectangular prototype filter used in cyclic prefix-OFDM (CP-OFDM). The W-OFDM waveform is generated by cyclic extension of the CP-OFDM signal followed by time domain windowing and overlap addition over time with the next symbol. Unlike f-OFDM, the filtering using $f(n)$ is bypassed. The per-subcarrier filtering with $p(n)$ is effectively carried out by time-domain windowing using a pulse $w(n)$ with smooth transition at the edges such that the response is localized with sharper decay in frequency domain. A typical window function is given by

$$
w(n)=\left\{\begin{array}{l}
0.5\left(1+\cos \left\{\pi\left(1+\frac{n}{N_{\beta}}\right)\right\}\right), 0 \leq n<N_{\beta} \\
1, N_{\beta} \leq n<N_{T} \\
0.5\left(1+\cos \left\{\pi \frac{n-N_{T}}{N_{\beta}}\right\}\right), N_{T} \leq n<N_{\beta}+N_{T}-1
\end{array}\right.
$$

$$
N_{T}=N+N_{C P}, N_{\beta}=\beta N_{T}
$$

Here $N_{C P}$ and $\beta$ represents the CP length and roll-offfactor which controls the roll-off portion of the window. At the receiver, the cyclic prefix is discarded and the signal is weighted by receiver windowing along edges. This is followed by overlap and addition. The detailed implementation aspects are provided in [6], [7].

\section{Design CONSTRAINTS AND CHALLENGES IN SATELLITE-TERRESTRIAL INTEGRATION}

The major design challenges and adaptations of 5G-PHYlayer standards, for its applicability in the integrated network scenario, are explained in this section.

\section{A. Non-linear Distortion by HPA and pre-distortion}

A major impairment that accounts for the poor performance of the OFDM based waveforms is the highly non-linear behaviour of the high power amplifiers (HPA) employed in satellites. Waveforms with large PAPR are more vulnerable to non-linear distortions that are irrecoverable at the receiver and lead to large performance degradations. A variety of techniques have been proposed in the literature for compensation of these degradations, among which, inverse-model based digital pre-distortion techniques can be implemented easily and cost effectively [16].

The output $v$ of HPA to an input signal $u=a e^{j \theta}$, can be expressed as

$$
v=T(a) e^{j((\theta)+\phi(a))}
$$

where $T(a)$ and $\phi(a)$ are the transformation functions corresponding to the amplitude/amplitude (AM/AM) and amplitude/phase (AM/PM) input-output characteristics of the HPA respectively. Amongst the HPAs used on board satellites, travelling wave tube amplifiers (TWTA) are highly non-linear in comparison to solid state power amplifiers (SSPA). The amplitude and phase input-output characteristics of a typical satellite TWTA provided by European Space Agency is used in the present investigation. The AM/AM and AM/PM characteristics are shown in Fig. 2. The characteristics can be modelled according to the generic memoryless modified Saleh model [17] which uses 6 parameters. According to the model [17], $T(a)$ is defined as

$$
T(a)=\frac{\alpha a^{\eta}}{\left(1+\beta a^{\gamma}\right)^{\nu}}+\epsilon
$$


TABLE I

TWTA SAleh Model Parameters.

\begin{tabular}{|l|l|l|}
\hline & $T(a)$ & $\phi(a)$ \\
\hline$\alpha$ & 4.08 & 4.107 \\
\hline$\beta$ & 2.456 & 5.203 \\
\hline$\epsilon$ & 0.0524 & -0.02394 \\
\hline$\gamma$ & 1.607 & 1.949 \\
\hline$\eta$ & 1.354 & 2.244 \\
\hline$\mu$ & 1.18 & 0.9249 \\
\hline
\end{tabular}

The different parameters for the AM/AM and AM/PM characteristics of the TWTA characteristics can be obtained using LS optimization procedure explained in [17]. For the present study, the parameters are obtained using curve fitting toolbox of MATLAB. The parameters are given in Table I. The variation of $T(a)$ and $\phi(a)$ modelled using the parameters in Table I are compared with the actual AM/AM and AM/PM characteristics in Fig. 2.

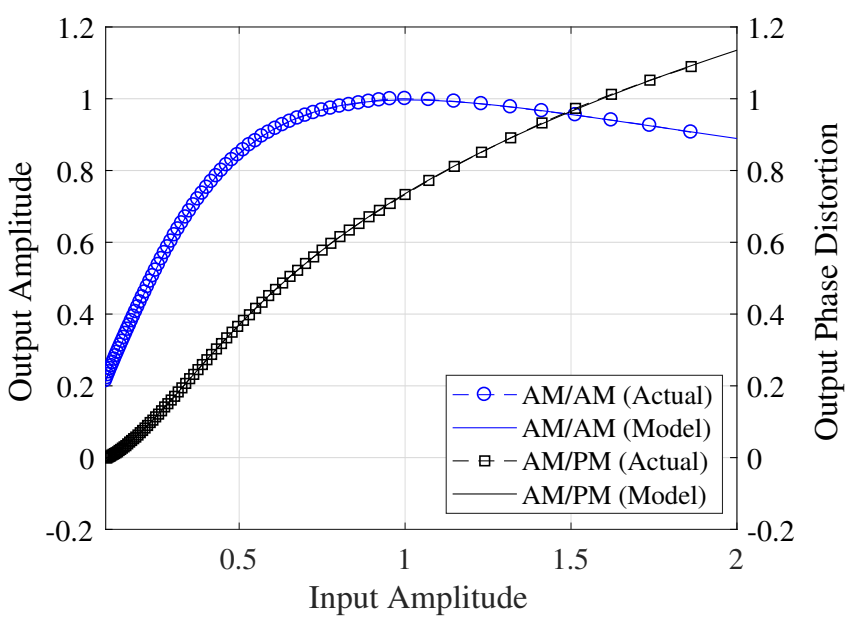

Fig. 2. Variation of $T(a)$ and $\phi(a)$ versus $a$ according to (6)

In a non-linear transmission scenario, both magnitude and phase needs to be pre-corrected. The modified phase of the output is the sum of the phase angle of the input and the corresponding phase distortion, $\phi(a)$. Hence, $\phi(a)$ can be precalculated according to the model for appropriate values of the input magnitude $a$ and is subtracted from the original phase of the signal before input to the TWTA. Unlike the phase variation, the amplitude distortion due to the TWTA is a non-linear operation. Hence, a pre-distortion method represented by an inverse function $T^{-1}(b)$ should be found such that $T\left(T^{-1}(a)\right)=a$. A straight forward inverse function formulation of the six parameter model given by (6), is intractable. However, if the input magnitude is limited to the monotonically increasing range, a simplified model derived from (6) with fewer parameters can be used to approximate the AM/AM characteristic which is given as

$$
T(a)=\frac{\alpha a^{\eta}}{\left(1+\beta a^{\eta}\right)}+\epsilon
$$

An inverse function of $T^{-1}(b)$ can be derived from (7) as

$$
T^{-1}(b)=\exp \left(\frac{1}{\eta} \ln \left(\frac{(b-\epsilon)}{\alpha-\beta(b-\epsilon)}\right)\right)
$$

In order to ensure the feasibility of the inverse operation, the operating point of the amplifier is backed-off away from the saturation point which is characterised by an input power back off (IBO) value. Hence, the magnitude of the transmitted signal $a$ is modified as $\tilde{a}=\lambda a$ where $\lambda<1$ is the reduction in magnitude corresponding to the IBO value. This can later be compensated at the receiver. The modified pre-compensated signal which is fed into the TWTA is given by

$$
\tilde{v}=T^{-1}(\tilde{a}) e^{j\left((\theta)-\phi\left(T^{-1}(\tilde{a})\right)\right)}
$$

The output of TWTA to $\tilde{v}$ is

$$
\tilde{u}=T\left(T^{-1}(\tilde{a})\right) e^{j\left((\theta)-\phi\left(T^{-1}(\tilde{a})\right)+\phi\left(T^{-1}(\tilde{a})\right)\right)}=\tilde{a} e^{j((\theta))}
$$

At the receiver, the transmitted signal can be compensated by multiplying with $\frac{1}{\lambda}$ so that $\frac{1}{\lambda} \tilde{u}=u$, which is the transmitted signal.

\section{B. Power limited link budget}

Satellite-terrestrial integration endeavours to maximise throughput for a given transmit power and service availability under adverse channel conditions. In order to maximise throughput and power efficiency, the operating point of the power amplifier should be set as close as possible to the saturation point. The operating point is decided by the IBO value which is defined as

$$
\mathrm{IBO}_{d B}=10 \log _{10}\left(\frac{P_{i n, \max }}{P_{\text {in }}}\right)
$$

where $P_{i n, \max }$ and $P_{i n}$ correspond to the maximum input power to the actual carrier power respectively. This corresponds to a reduction in the output power which is characterised by the output back-off (OBO) defined by the ratio of maximum output saturation power $P_{\text {out }, \max }$ to the output power $P_{\text {out }}$ as

$$
\mathrm{OBO}_{d B}=10 \log _{10}\left(\frac{P_{\text {out }, \text { max }}}{P_{\text {out }}}\right)
$$

This will depend on the actual link budget for the specific user carrier and its required QoS. The AM/AM non-linear distortion characteristics curve in Fig. 2 can be divided into three regions. The linear region at very low input magnitude levels, non-linearly distorted region at input magnitude levels close to saturation point, and the region corresponding to input levels greater than the saturation point. Operating the amplifier at very low input amplitude levels (IBO, OBO $>>0$ ) can reduce the non-linear distortion to a great extent but at the expense of reduced power amplifier efficiency. Ideal operating point with respect to amplifier efficiency is with $\mathrm{OBO}=0$ but the distortion due to non-linear clipping for magnitude levels greater than the saturation value are irrecoverable and cannot be corrected with pre-distortion (PD) techniques. Hence, 
operating the power amplifier, near the saturation point is a desirable solution which maximises efficiency and ensures the feasibility of linearisation by PD techniques. This demands the candidate waveform to have low PAPR and very low SNR operating points.

\section{Mobility of the transmission/user equipment}

For non-geostationary satellites, the relative motion between the receiver and the transmitter create large Doppler shift. The situation is degraded when the user equipment is located within high speed trains or air-craft, where, the maximum speeds can reach to $1000 \mathrm{~km} / \mathrm{hr}$. This corresponds to a very large Doppler shift especially in the case of low earth orbit (LEO) satellites in $\mathrm{Ka}$ band where the maximum Doppler shift is $\pm 480 \mathrm{kHz}$ at $\pm 5.44 \mathrm{kHz} / \mathrm{s}$ variation rate. The mobility can also introduce extra complexity in beam management for efficient handover because of the moving cell pattern. The transmission delay is large for satellite-terrestrial communication. The mobility leads to a change in the overall distance and hence the transmission delay becomes a variable quantity. This demands dynamic timing-advance alignment strategies at the receiver.

Even though mobility creates the aforementioned challenges, the Doppler shift pre-compensation, dynamic beam management, handover and timing advance alignment can be simplified with the knowledge of user location and satellite ephemeris [3], [18]. Phase variation in the time domain due to uncompensated Doppler shifts, residual carrier frequency offset and phase noise due to imperfect local oscillator performance are still challenges in the integrated satellite-terrestrial scenario and hence need more research attention.

\section{NumericAl ANALYSis}

In this section, we present the performance evaluation of the candidate waveforms under satellite channel impairments using relevant metrics such as total degradation at target BER, OOB emission based on spectral analysis, PAPR etc. The FFTsize and the cyclic prefix length are chosen as $N=1024$ and $N_{C P}=72$ respectively. The roll-off ratio for the transmit and receive windows for W-OFDM are selected as $4 \%$ and $1 \%$ respectively. The signal is modulated using 16-QAM. The inverse function (8) is used for performing the PD operation as explained in Section III-A. The curves corresponding to the actual AM/AM characteristics, $T(a), T^{-1}(a)$ and $T\left(T^{-1}(a)\right)$ are shown in Fig. 3. From the figure, it can be inferred that, for the monotonically increasing range of $a$, the simplified model approximates the actual AM/AM characteristic curve and the TWTA distortion is linearised by the inverse function. The parameters of the simplified model (7) are $\alpha=11.38$, $\beta=11.95, \eta=2.058$ and $\epsilon=0.1385$.

\section{A. Impact of non-linear distortion by HPA}

The robustness of the candidate waveform against nonlinear HPA distortion mainly depends on the PAPR of the waveform. The PAPR evaluation is carried out using the complementary cumulative distribution function (CCDF) which

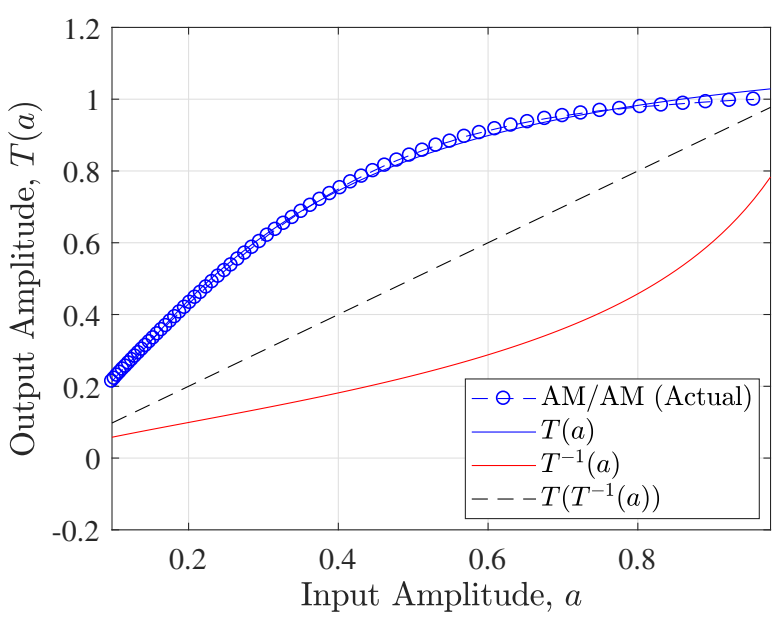

Fig. 3. Variation of actual AM/AM characteristics, $T(a), T^{-1}(a)$ and $T\left(T^{-1}(a)\right)$ versus $a$

denotes the probability that the PAPR is greater than a specified value. The CCDF versus PAPR plots of the waveforms are compared in Fig. 4. DFT-s-W-OFDM and DFT-s-f-OFDM has lower PAPR values of $2.7 \mathrm{~dB}$ and $3.85 \mathrm{~dB}$ at $\mathrm{CCDF}=10^{-3}$ and suffers less distortion due to the TWTA amplifier. The f-OFDM and W-OFDM have similar PAPR values that are greater than $11 \mathrm{~dB}$.

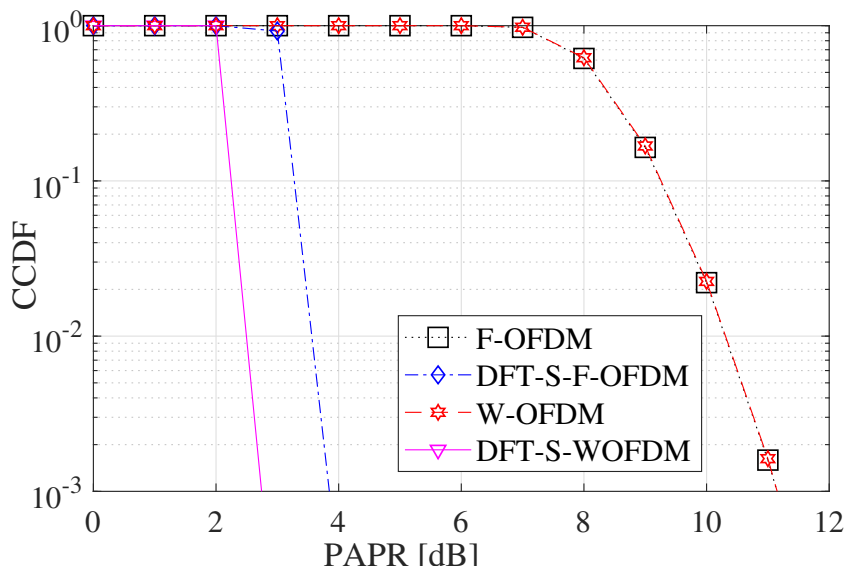

Fig. 4. PAPR Comparison

The OOB emission of the waveforms under non-linear TWTA distortion are compared using power spectral density (PSD) plots and are presented in Fig. 5. The PSDs of noiseless original waveforms, non-linearly distorted waveforms with and without $\mathrm{PD}$ at $\mathrm{OBO}=3 \mathrm{~dB}$ are illustrated. The $\mathrm{OOB}$ emission of the candidate waveforms are below $-50 \mathrm{~dB}$ in the absence of TWTA non-linearity. The spectral distortion due to the TWTA is evident for all the waveforms without PD operation but is successfully reduced with PD. The DFT-s-W-OFDM which has the lowest PAPR shows the best OOB suppression. It is worth noting that the PSD of pre-distorted DFT-s-W-OFDM, whose PAPR is comparable to the used OBO value, coincides 


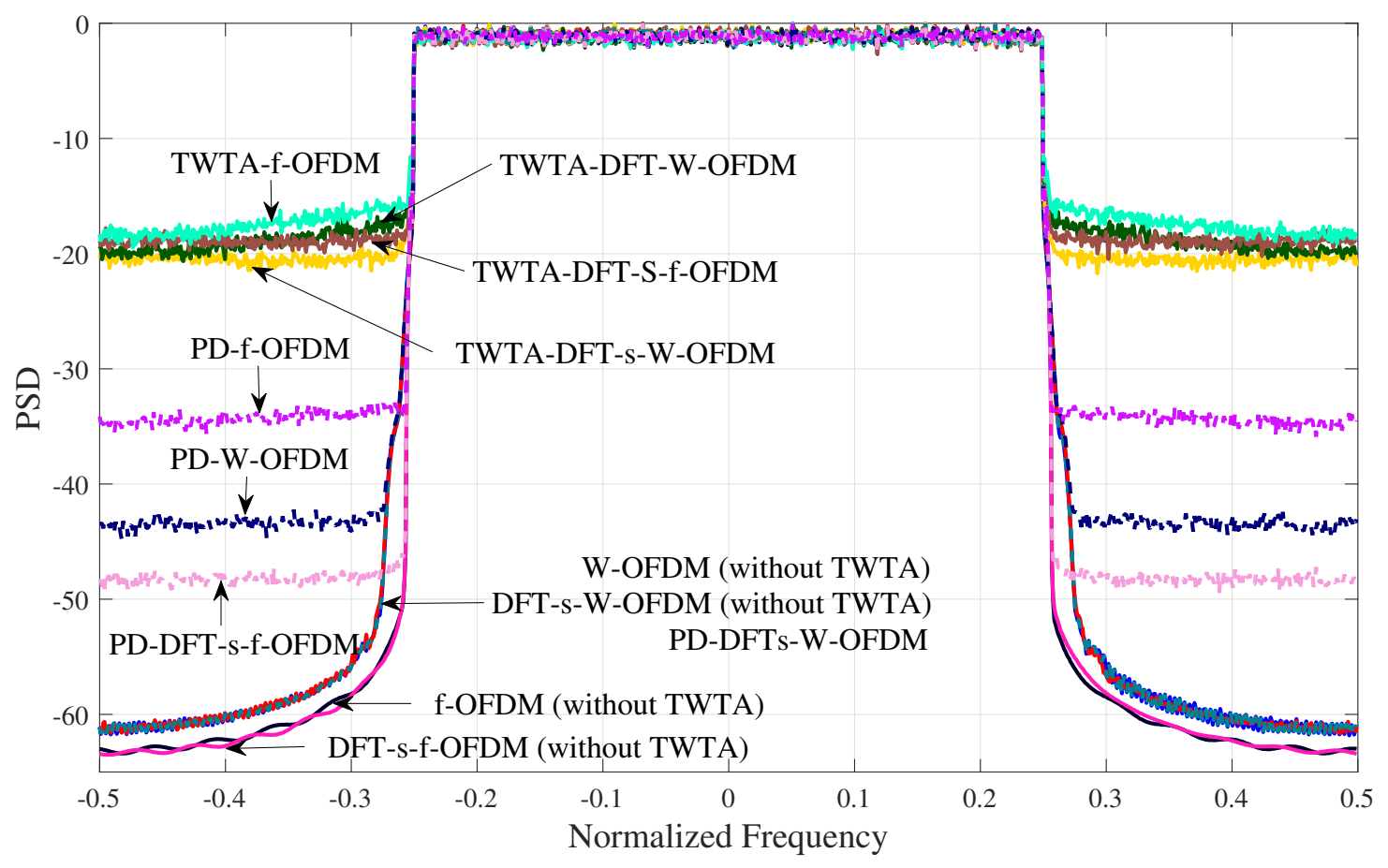

Fig. 5. PSD Comparison for $\mathrm{OBO}=3 \mathrm{~dB}$

with that of the noiseless original W-OFDM and DFT-s-WOFDM waveforms.

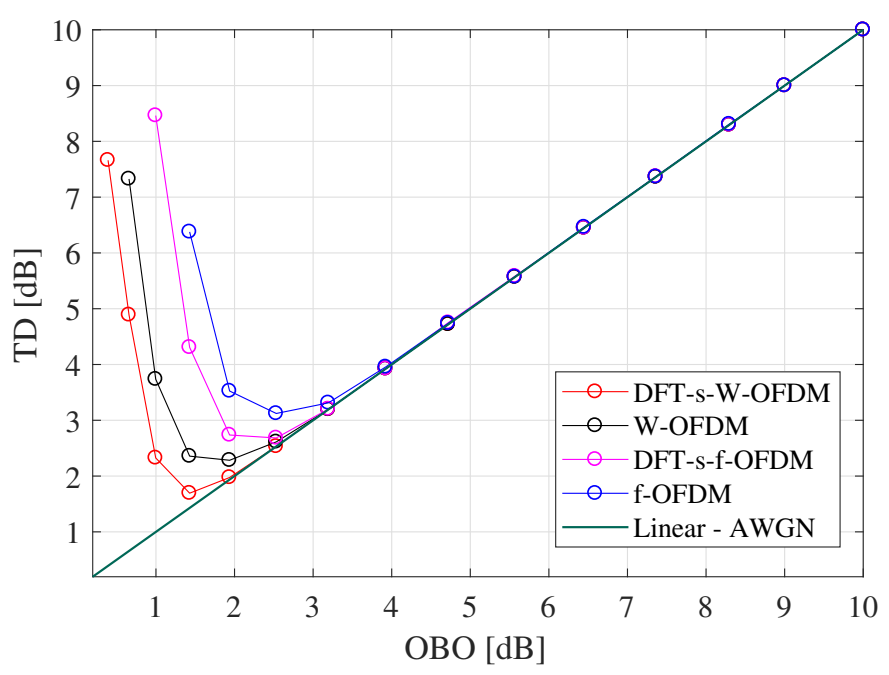

Fig. 6. Total Degradation versus OBO

A distinctive metric for quantifying the impact of non-linear TWTA distortion is the total degradation (TD) which is a function of OBO. The total degradation is defined as

$$
\mathrm{TD}_{\mathrm{dB}}=\mathrm{OBO}_{\mathrm{dB}}+\left.\frac{E_{b}}{N_{0}}\right|_{\mathrm{NL}}-\left.\frac{E_{b}}{N_{0}}\right|_{\mathrm{L}}
$$

TABLE II

S-BAND SATELLITE PHASE NOSIE MASK.

\begin{tabular}{|c|ccccccc|}
\hline $\mathrm{f}$ & $10 \mathrm{~Hz}$, & $100 \mathrm{~Hz}$, & $1 \mathrm{kHz}$, & $10 \mathrm{kHz}$, & $100 \mathrm{kHz}$, & $1 \mathrm{MHz}$, & $10 \mathrm{MHz}$ \\
\hline $\mathrm{dBc} / \mathrm{Hz}$ & -29, & -59, & -69, & -74, & -83, & -95, & -101 \\
\hline
\end{tabular}

where $\left.\frac{E_{b}}{N_{0}}\right|_{N L}$ and $\left.\frac{E_{b}}{N_{0}}\right|_{L}$ are the per-bit signal to noise ratio (SNR) required to maintain a target bit error rate (BER) under non-linear distortion due to the actual TWTA and a perfectly linear TWTA respectively. The TD versus OBO performance comparison for an un-coded system with a target BER of $10^{-4}$ is presented in Fig. 6. A linear curve which corresponds to additive white gaussian noise conditions without TWTA is also shown for comparison. DFT-s-W-OFDM shows the best TD performance which has the least distortion at minimum OBO value of $1.5 \mathrm{~dB}$. The minimum TD occurs at $1.95 \mathrm{~dB}, 2.5$ $\mathrm{dB}$ and $2.52 \mathrm{~dB}$ for W-OFDM, DFT-s-f-OFDM and f-OFDM respectively.

\section{B. Impact of CFO, Phase Noise and Multipath Channel}

In this section, the performance of the waveforms is evaluated under integrated-satellite-terrestrial multipath channel, CFO and phase noise. Half-rate convolutional coding is used for channel coding. We use the NTN-tapped delay line (TDL) channel model [3] as the multipath channel model. The parameters of the S-band satellite phase-noise mask [19] used for the simulation of phase noise is given in Table II. The BER versus SNR performance comparison of the candidate waveforms for $\mathrm{CFO}=0.1$ at $\mathrm{OBO}=3 \mathrm{~dB}$ is presented in 
Fig. 7. The plot corresponding to $\mathrm{CFO}=0$ is also shown for comparison. Simple one-tap zero-forcing equalization is performed to reduce multipath-distortions. The f-OFDM based waveforms shows better BER performance than W-OFDM and its variants. In the absence of CFO at high SNRs, the f-OFDM and W-OFDM shows better performance than their DFT spread variants.

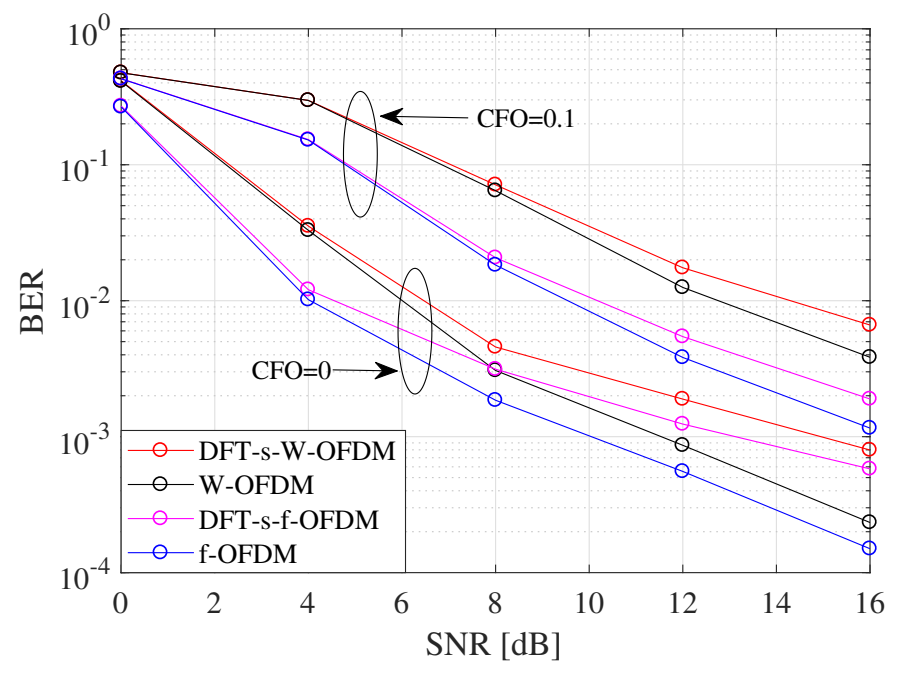

Fig. 7. BER versus SNR

\section{CONClusion}

In this paper, we have investigated the adaptability of OFDM based candidate waveforms recommended by 3GPP for NR, in an integrated satellite-terrestrial scenario. The performance of the NR candidate waveforms were analysed under the impact of main impairments in the integrated scenario such as the high non-linear distortion by TWTA, NTN-multipath channel, $\mathrm{CFO}$ and phase noise based on various metrics and simulation parameters defined by 3GPP for the NR to support NTN. An inverse function based pre-distortion method is presented and applied to minimize the non-linear TWTA distortion. Simulation results conform that, with appropriate predistortion and channel equalization techniques, the W-OFDM and f-OFDM waveforms that are already recommended for use in 3GPP-NR, are promising candidates for air interface over satellite channels. The DFT spread versions of the candidate waveforms that have lower PAPR also have smaller OOB emission than their counterparts. The W-OFDM based waveforms have smaller total-degradation than the f-OFDM based waveforms at the same OBO under non-linear distortion of the HPA. In the presence of CFO, non-linearity, multipath and phase noise, BER analysis showed that, the f-OFDM based waveforms outperform W-OFDM based waveforms. CFO estimation and correction along with phase noise mitigation can be considered for further studies.

\section{ACKNOWLEDGMENT}

This work was sponsored by China Academy of Space Technology (CAST)-Xian. The authors also would like to acknowledge the support of the University of Surrey 5GIC (http://www.surrey.ac.uk/5gic).

\section{REFERENCES}

[1] A. Guidotti, A. Vanelli-Coralli, M. Conti, S. Andrenacci, S. Chatzinotas, N. Maturo, B. Evans, A. Awoseyila, A. Ugolini, T. Foggi, L. Gaudio, N. Alagha, and S. Cioni, "Architectures and key technical challenges for 5G systems incorporating satellites," IEEE Transactions on Vehicular Technology, vol. 68, no. 3, pp. 2624-2639, March 2019.

[2] K. Liolis, A. Geurtz, R. Sperber, D. Schulz, S. Watts, G. Poziopoulou, B. Evans, N. Wang, O. Vidal, B. Tiomela Jou et al., "Use cases and scenarios of $5 \mathrm{G}$ integrated satellite-terrestrial networks for enhanced mobile broadband: The SaT5G approach," International Journal of Satellite Communications and Networking, vol. 37, no. 2, pp. 91-112, 2019.

[3] 3GPP, "Study on New Radio (NR) to support non-terrestrial networks," 3rd Generation Partnership Project (3GPP), Technical Report (TR) RAN-80, 08 2018, version 15.0.0.

[4] J. Abdoli, M. Jia, and J. Ma, "Filtered OFDM: A new waveform for future wireless systems," in 2015 IEEE 16th International Workshop on Signal Processing Advances in Wireless Communications (SPAWC), June 2015, pp. 66-70.

[5] L. Zhang, A. Ijaz, P. Xiao, M. M. Molu, and R. Tafazolli, "Filtered OFDM systems, algorithms, and performance analysis for $5 \mathrm{G}$ and beyond," IEEE Transactions on Communications, vol. 66, no. 3, pp. 1205-1218, March 2018.

[6] R. Zayani, Y. Medjahdi, H. Shaiek, and D. Roviras, "WOLA-OFDM: A potential candidate for asynchronous 5G," in 2016 IEEE Globecom Workshops (GC Wkshps), Dec 2016, pp. 1-5.

[7] X. Zhang, L. Zhang, P. Xiao, D. Ma, J. Wei, and Y. Xin, "Mixed numerologies interference analysis and inter-numerology interference cancellation for windowed OFDM systems," IEEE Transactions on Vehicular Technology, vol. 67, no. 8, pp. 7047-7061, Aug 2018.

[8] B. Farhang-Boroujeny, "OFDM versus filter bank multicarrier," IEEE Signal Processing Magazine, vol. 28, no. 3, pp. 92-112, May 2011.

[9] L. Zhang, P. Xiao, A. Zafar, A. u. Quddus, and R. Tafazolli, "FBMC system: An insight into doubly dispersive channel impact," IEEE Transactions on Vehicular Technology, vol. 66, no. 5, pp. 3942-3956, May 2017.

[10] G. Fettweis, M. Krondorf, and S. Bittner, "GFDM - generalized frequency division multiplexing," in VTC Spring 2009 - IEEE 69th Vehicular Technology Conference, April 2009, pp. 1-4.

[11] J. Zhong, G. Chen, J. Mao, S. Dang, and P. Xiao, "Iterative frequency domain equalization for MIMO-GFDM systems," IEEE Access, vol. 6, pp. 19386-19395, 2018.

[12] V. Vakilian, T. Wild, F. Schaich, S. ten Brink, and J. Frigon, "Universalfiltered multi-carrier technique for wireless systems beyond LTE," in 2013 IEEE Globecom Workshops (GC Wkshps), Dec 2013, pp. 223228.

[13] L. Zhang, A. Ijaz, P. Xiao, A. Quddus, and R. Tafazolli, "Subband filtered multi-carrier systems for multi-service wireless communications," IEEE Transactions on Wireless Communications, vol. 16, no. 3, pp. 1893-1907, March 2017.

[14] Y. Medjahdi, Y. Louet, M. B. Mabrouk, D. Roviras, R. Zakaria, H. Shaiek, D. L. Ruyet, S. Traverso, R. Gerzaguet, D. Demmer, J. Dore, and R. Zayani, "Impact of selective channels on post-OFDM waveforms for 5G machine type communications," in 2018 15th International Symposium on Wireless Communication Systems (ISWCS), Aug 2018, pp. $1-5$.

[15] 3GPP, "OFDM based flexible waveform for 5G," 3rd Generation Partnership Project (3GPP), Discussion RAN1-84 BIS, 04 2016, R1-162152.

[16] H. I. Durney Wasaff, Adaptive pre-distortion for nonlinear high power amplifiers in OFDM systems. Universitat Politècnica de Catalunya, 2004.

[17] M. O'droma, S. Meza, and Y. Lei, "New modified saleh models for memoryless nonlinear power amplifier behavioural modelling," IEEE Communications Letters, vol. 13, no. 6, pp. 399-401, 2009.

[18] 3GPP, "Considerations on Doppler compensation for Non-GEO-based NTN," 3rd Generation Partnership Project (3GPP), Discussion and decision RAN1-\#96, 04 2019, R1-1904245.

[19] — "Key satellite parameters and simulation assumptions for NTN," 3rd Generation Partnership Project (3GPP), Discussion RAN1-96, 04 2019, R1-1905216. 